\title{
Monitoring the Prevalence of Metabolic Syndrome Among Hypothyroid Patients and Assessing the Effect of Anti-Hypothyroid Treatment on it Among the South Indian Population
}

\author{
Balasubramanian Ganesh Pandian ${ }^{1 *}$, Paruchuri Sireesha², Ng Yen Ping ${ }^{1}$, Nagula Parashuram ${ }^{3}$ \\ 'Unit of Clinical Pharmacy and Pharmacy Practice, AIMST University, Semeling, MALAYSIA. \\ ${ }^{2}$ Department of Pharmacy Practice, Vaagdevi College of Pharmacy, Warangal, INDIA. \\ 'Department of ENT, Kakatiya Medical College/Mahatma Gandhi Memorial Hospital, Warangal, INDIA.
}

\begin{abstract}
Objective: Hypothyroidism and metabolic syndrome are well associated risk factors for atherogenic cardiovascular diseases. This study was intended to investigate the prevalence of metabolic syndrome and to assess its related parameters in hypothyroid patients. Material and Methods: Study was conducted in ENT department of a tertiary care teaching hospital. Thirty patients diagnosed with hypothyroidism of age $12-40$ years of both sexes were screened for metabolic and thyroid parameters. Among them, who were diagnosed with metabolic syndrome according to IDF criteria were included in the study. The baseline patients were treated with L-thyroxin and reviewed after 3 months. Baseline and reviewed parameters were compared. Data were analyzed with paired ' $t$ ' test. Results: The mean age of the study population was found to be $26.9 \pm 8.1$ years and among the study population $96.66 \%$ were female. Among the study population $(n=30), 13$ patients were diagnosed with metS according to the IDF criteria and data from these patients was used as the baseline, after L-Thyroxine treatment for three months it was found that around $70 \%$ were out of metS. Statistical result shows the significant changes in metabolic parameters with treatment. Further the association of metabolic and thyroid parameters were also found. Conclusion: Present study shows that the female have higher prevalence of hypothyroidism. Further study results show significant association between hypothyroidism and metabolic
\end{abstract}

syndrome which necessitates the need for monitoring thyroid functions in patients with metabolic syndrome.

Key words: Hypothyroidism, L-Thyroxine, Metabolic syndrome, metS, Sub Clinical Hypothyroidism.

\section{PICTORIAL ABSTRACT}

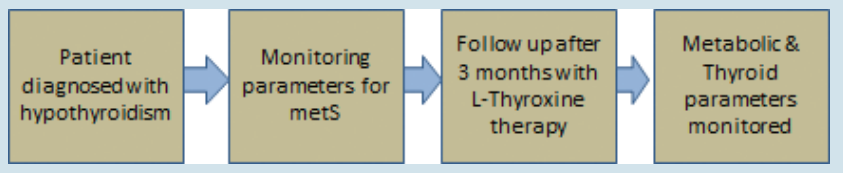

Correspondence:

Balasubramanian Ganesh Pandian,

Unit of Clinical Pharmacy and Pharmacy Practice, AIMST University, Semeling, MALAYSIA.

Phone no: +6044298000

E-mail: ganeshhhh1@gmail.com

DOI: 10.5530/jyp.2016.2.8

\section{INTRODUCTION}

Metabolic syndrome (metS) and hypothyroidism are well-established precursor of atherogenic cardiovascular disease. Hypothyroidism is well known to cause endothelial dysfunction, hyperlipidaemia, cardiovascular disease and diastolic hypertension. ${ }^{1}$ Significant overlap exists in the pathogenic mechanisms of atherosclerotic cardiovascular disease by metS and hypothyroidism. ${ }^{2}$ A study on thyroid dysfunction in metS population may help in knowing the extent of overlap between these two groups and may emphasize the importance of thyroid function tests (TFT) in identifying hypothyroid population from metS.

This can result in proper planning and suitable management strategies, resulting in notable reduction in cardiovascular mortality and morbidity due to metS by effectual thyroid replacement therapy. Metabolic syndrome has been linked to subclinical thyroid disease in older adults due to pathophysiology of thyroid function on lipid glucose metabolism, blood pressure, and cardiovascular dysfunction. ${ }^{3}$ Increased likelihood of metS occurrence is associated with high TSH levels, especially in females (which concurrently exists with overt hypothyroidism) increases the risk of cardiovascular disease (CVD). ${ }^{4}$ Incidence of subclinical hypothyroidism is more among female with metS, ${ }^{5}$ the reports of HYOGA study shows that: even when the TSH levels were less than $10 \mathrm{mlIU} / \mathrm{L}$ symptoms of hypothyroidism and poor QOL was reported among hypercholestrolaemic female with SCT and over 50 years of age. ${ }^{6}$ Both metabolic and thyroid parameters affect the quality of life (QOL) of patients with thyroid dysfunction. ${ }^{7}$ High TSH levels are associated with harmful changes in serum lipids among older white women. ${ }^{8}$ The thyroid hormones up-regulate the metabolic pathways pertinent to resting energy expenditure, and hence, obesity and thyroid functions are usually compared..$^{9}$ On the other hand, obesity itself prompt alterations in thyroid hormones ${ }^{10}$ increased TSH with no influence on $\mathrm{T}_{3}$ (Tri-iodo thyronine) and $\mathrm{T}_{4}$ (Thyroxin) ${ }^{11}$ or increase in $\mathrm{TSH}$ and $\mathrm{T}_{3}$ without affecting $\mathrm{T}_{4}$; further, subclinical hypothyroidism as a result of prolonged metabolism which leads to obesity. ${ }^{12}$

The standard levels of $\mathrm{T}_{3}, \mathrm{~T}_{4}$ with elevated TSH in metS is not defined, but it has been guesstimated that metS is related with insulin resistance due to the deficiency in post receptor signal transduction in target tissue; a homogeneous mechanism of thyroid receptor resistance might function in these persons. ${ }^{11}$

The present study was conducted to assess the prevalence of metabolic syndrome among the patients with hypothyroid disorders and its changes with anti-thyroid treatment among the south Indian population.

\section{MATERIALS AND METHODS}

This is a prospective observational study conducted at the out-patient department of ENT and Endocrinology, Mahatma Gandhi Memorial Hospital, Warangal which is a 1000 bedded multidisciplinary government and tertiary care teaching hospital. The study was carried out for a period of seven months. This study was approved by the Institutional Ethics committee and all the data were collected according to the hospital regulations. Patients aged 12 to 40 years diagnosed with hypothyroidism 
for the first time were included in the study irrespective of any gender. The study population consisted of 30 diagnosed hypothyroid cases; all patients were diagnosed hypothyroid based on the supporting clinical laboratory investigations of hypothyroidism (increased TSH, decreased $\mathrm{tT}_{3}$ and $\mathrm{tT}_{4}$ ). The patients were also sorted for different epidemiological factors like age, gender, marital status, socio-economic status, representative area (Rural/urban) and life style pattern. Informed consent was obtained from all the patients by explaining about the study and the patients those who shown interest to participate only were included in our study. Known hypothyroid patients and patients on lipid lowering agents, radio-active treatment, pregnant and lactating women were not included in this study. All the patients were advised for metabolic parameters which includes waist circumference (WC), Body Mass index (BMI), fasting blood sugar (FBS), high density lipoproteins (HDL), triglycerides (TG). All the data were collected from the respective patient's medical chart. Among the study patients thirteen were diagnosed as having metabolic syndrome based on the International Diabetes Federation (IDF) criteria. These patients taken as baseline and all of them were treated with L-thyroxin 25 and $50 \mathrm{mcg} /$ day (depending upon the severity of hypothyroidism) in an empty stomach before breakfast daily for 3 months and these patients were asked to come for review after 3 months and again advised for thyroid and metabolic parameters. Baseline and review parameters were compared. The parameters monitored were statistically analysed with paired ' $\mathrm{t}$ ' test using Graph pad prism version-6.

\section{RESULTS}

The study group includes 30 hypothyroid patients with 29 female and 1 male. The mean age of the study population was $26.9 \pm 8.1$ years. Among the study population $66.6 \%$ were diagnosed as subclinical hypothyroidism and $33.3 \%$ were overt hypothyroidism. According to the IDF criteria the study population were monitored for the metabolic syndrome, 13 patients were found to have metabolic syndrome. According to the parameters among the study population monitored during baseline treatment it was found that 13 patients were having higher waist circumference (WC) than the normal, nine were having higher blood pressure (BP), seven were having increased fasting blood sugar (FBS) levels, twelve were having low high density lipoprotein cholesterol (HDLc) levels and none were having increased triglyceride (TG) levels. Average (mean $\pm \mathrm{SD}$ ) values of thyroid parameters measured at baseline were found to be for thyroid stimulating hormone (TSH) as $51.9 \pm 61.1$, triiodothyronine $\left(\mathrm{tT}_{3}\right)$ as $96.9 \pm 43.07$ and tetraiodothyronine $\left(\mathrm{tT}_{4}\right) 5.7 \pm 2.88$. Patients were undergoing treatment with antithyroid medicine, the metabolic and thyroid parameters were monitored 3 months later after starting the therapy (baseline). It was noted that

Table 1: Comparison of Baseline and Review parameters

\begin{tabular}{cccc}
\hline Parameters & $\begin{array}{c}\text { Baseline } \\
(\text { mean } \pm \text { SD })\end{array}$ & $\begin{array}{c}\text { Review } \\
(\text { mean } \pm \text { SD) }\end{array}$ & P-Value $^{\text {s }}$ \\
\hline BMI $\left(\mathrm{kg} / \mathrm{m}^{2}\right)$ & $21.01 \pm 3.61$ & $22.6 \pm 4.91$ & $0.0002^{* *}$ \\
WC $(\mathrm{cm})$ & $87 \pm 11.5$ & $92.4 \pm 9.13$ & 0.2676 \\
Systolic BP $(\mathrm{mmHg})$ & $114.55 \pm 10.34$ & $119.2 \pm 11.15$ & $0.0197^{*}$ \\
Diastolic BP $(\mathrm{mmHg})$ & $79.3 \pm 8.27$ & $76.68 \pm 8.54$ & 0.0950 \\
Blood sugar $(\mathrm{mg} / \mathrm{dL})$ & $93.4 \pm 17$ & $82.74 \pm 6.4$ & $0.0472^{*}$ \\
HDLc $(\mathrm{mg} / \mathrm{dL})$ & $47.4 \pm 6.42$ & $42 \pm 10.8$ & 0.2141 \\
Triglyceride $(\mathrm{mg} / \mathrm{dL})$ & $90.8 \pm 18$ & $88 \pm 24$ & 0.8413 \\
TSH $(\mu \mathrm{IU} / \mathrm{L})$ & $51.9 \pm 61.1$ & $3.4 \pm 1.13$ & $0.0169^{*}$ \\
tT3 $(\mathrm{ng} / \mathrm{dL})$ & $96.9 \pm 43.07$ & $119 \pm 11.6$ & 0.1976 \\
tT4 $(\mu \mathrm{g} / \mathrm{dL})$ & $5.7 \pm 2.88$ & $7.7 \pm 1.54$ & 0.1455 \\
\hline
\end{tabular}

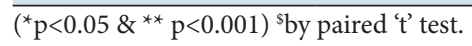

the clinical parameters for metS were found to be normal in majority of the patients, after treatment among 13 patients with metS during baseline 10 were recovered from metS. The clinical parameters monitored during the baseline and review after 3 months of L-thyroxin therapy was analyzed statistically which show a clinically significant difference in the parameters as BMI, BP, Blood sugar levels and TSH levels with the L-Thyroxine therapy as mentioned in Table 1. Further, the monitored metabolic and thyroid parameters were analysed for their association over the other. The results obtained were given in Table 2.

\section{DISCUSSION}

Metabolic syndrome is a cluster of cardiovascular risk factors and it is characterized by inflammation. ${ }^{5}$ It was reported that $60 \%$ of cases had thyroid function abnormalities in their study. ${ }^{13}$ Among the study population, the prevalence of metabolic syndrome and its consequences related to thyroid dysfunction is more in female and earlier studies shows that the thyroid dysfunction was common in females with metS.,14 $66 \%$ of the study population presented with subclinical hypothyroidism and the rest with overt hypothyroidism, previous studies reported a high prevalence of subclinical hypothyroidism among female with metabolic syndrome. ${ }^{5} 15$ to 35 years of age had a high incidence of thyroid dysfunction as compared to other age groups with the mean age of $26.9 \pm 8.1$ years, where age of 40 to 60 years had a high incidence of thyroid dysfunction as compared to the other groups. ${ }^{4}$

The association between dyslipidaemia and thyroid hypo function is well established. Thyroid hormone is well known to play a role in balancing the synthesis, metabolism, and mobilization of lipids. In patients with overt hypothyroidism, there is an elevation in serum total cholesterol, LDL levels and possibly, triglyceride level. ${ }^{1}$ Normally, thyroid hormones raise the expression of cell surface of the LDL receptors, thus resulting in clearance of LDL from the serum. In hypothyroidism, the reduction of the thyroid hormones leads to decreased number of LDL receptors in the liver, thereby results in decreasing the biliary excretion of cholesterol, thus resulting in increased LDL and VLDL levels. It also reduces the lipoprotein lipase activity and causes hypertriglyceridaemia. ${ }^{13}$ A high LDL is not a component of metS instead reduced HDLc is a metabolic syndrome component and hence the causative role of thyroid hypofunction in the pathogenesis of metS needs to be investigated.

Study population who were on L-thyroxine for 3 months were checked for metabolic parameters and found only $23.07 \%$ with metS showing L-thyroxine's beneficial effect on metabolic parameters especially on serum lipids. Tromso Study and Basel Thyroid Study have shown that L-thyroxine replacement in patients with subclinical hypothyroidism has a beneficial effect on low density lipoprotein cholesterol levels and clinical symptoms of hypothyroidism.

The relationship between serum TSH levels and components of metS concluding that even slight increase in TSH as in subclinical hypothyroidism may be a metS risk factor. The main findings of this study indicate that lipid profile especially HDLc levels were more adversely affected by $\mathrm{SCH}$ and $\mathrm{OH}$ and therefore would more often result in metS. In our study, $92 \%$ of hypothyroid patients are having lower HDLc. In fact, only a high waist circumference and a low HDL were the only other components of the metS which were more common. The other components of metS like hypertension, hypertriglyceridaemia and glucose intolerance were less common than the cumulative incidence of thyroid function abnormalities. Thus the results of this study justify the routine measurement of thyroid function tests in patients with metS and vice-versa. Thus, the detection and treatment of thyroid function abnormalities in metS patients or measuring metabolic parameters in hypothyroid patients may contribute to the reduction of the increased 
PANDIAN, et al:: Metabolic Syndrome Among Hypothyroid Patients

Table 2: Association of metabolic parameters over thyroid parameters among the study population

\begin{tabular}{|c|c|c|c|c|c|c|}
\hline \multirow{2}{*}{ Parameter } & \multicolumn{2}{|c|}{ TSH } & \multicolumn{2}{|c|}{$T_{3}$} & \multicolumn{2}{|c|}{$T_{4}$} \\
\hline & Baseline $\left(p^{\#}\right)$ & Review ( $p^{\#)}$ & Baseline $\left(p^{\#)}\right.$ & Review $\left(\mathrm{p}^{\#)}\right.$ & Baseline $\left(p^{\#)}\right.$ & Review ( $p^{\#)}$ \\
\hline BMI & 0.4772 & 0.4177 & 0.7866 & 0.1787 & 0.9148 & 0.9085 \\
\hline WC & 0.7309 & 0.3877 & 0.2389 & 0.4654 & 0.6041 & 0.9627 \\
\hline Blood sugar & 0.7013 & 0.8890 & 0.8851 & 0.0982 & 0.7574 & 0.7066 \\
\hline HDLc & 0.1476 & 0.1092 & 0.3494 & 0.2475 & 0.3735 & 0.1823 \\
\hline Triglyceride & 0.2618 & 0.7208 & 0.5277 & 0.4702 & 0.5812 & 0.6541 \\
\hline Systolic BP & 0.3638 & 0.6860 & 0.7039 & 0.3596 & 0.9182 & 0.4250 \\
\hline Diastolic BP & 0.6700 & 0.6069 & 0.6714 & 0.3472 & 0.9590 & 0.8154 \\
\hline
\end{tabular}

$\left({ }^{*} \mathrm{p}<0.05 \&{ }^{* *} \mathrm{p}<0.001\right) ;$ \# is by Pearson's correlation.

cardiovascular or hypothyroid risk that those patients have. It is well proved in various studies that the metabolic syndrome is becoming an unavoidable factor in case of hypothyroidism and various studies stress to monitor the parameters for metS while diagnosing a patient as hypothyroid.

Being this study conducted with small sample size, significant association between the metabolic and thyroid parameters were not established. Where, a study with large sample size can establish the significant association between them.

\section{CONCLUSION}

Present study shows that the female with hypothyroidism have higher prevalence of metabolic syndrome. There occur is an interrelationship between thyroid function and cardiovascular risk factors such as blood sugar, BP, and HDLc which are the predisposing factors. This study concludes by revealing a high incidence of metS among thyroid dysfunc- tion especially sub-clinical hypothyroidism which undoubtedly supports the previous research conducted that there is a valid need to monitor the parameters for metS for the patients diagnosed with hypothyroidism. Regular screening for cardiovascular risk factors in patients with hypothyroidism may unmask metS and increased TSH levels may especially become a component of metabolic syndrome in future which further affects the patients living quality also.

\section{ACKNOWLEDGEMENT}

Authors would like to thank the Superintendent, MGM Hospital for permitting us to conduct this study and patients for actively participating in this study.

\section{CONFLICT OF INTEREST}

Authors declared there is no any conflict of interest.

\section{ABBREVIATIONS USED}

BMI: Body mass index; BP: Blood pressure; CVD: Cardio vascular disease; FBS: Fasting blood sugar; HDL: High density lipoprotein; IDF: International diabetes federation; LDL: Low density lipoprotein; metS: Metabolic syndrome; OH: Overt hypothyroidism; QOL: Quality of life; SCH: Subclinical hypothyroidism; T3: Triiodothyronine; T4: Tetraiodothyronine; TFT: Thyroid function tests; TG: Triglyceride; TSH: Thyroid stimulating hormone; VLDL: Very low density lipoprotein; WC: Waist circumference.

\section{ABOUT AUTHORS}

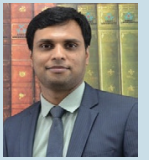

Balasubramanian Ganesh Pandian: Is currently a Lecturer in the Unit of Clinical Pharmacy and Pharmacy Practice, AIMST University, Malaysia. He graduated in Master of Pharmacy in Pharmacy from Tamilnadu Dr. M.G.R Medical University, Chennai, India. His area of expertise includes metabolic syndrome and its quality of life among them. He has published various research articles in journals.

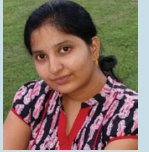

Sireesha Paruchuri: Is currently a Pharmacist graduated in Master of Pharmacy in Pharmacy Practice from Kakatiya University, Warangal, India. Her research interest includes metabolic syndrome and endocrinology. She had published research and review article in various journals.

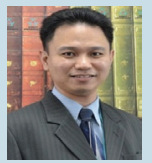

Ng Yen Ping: Is currently a clinical pharmacy lecturer in Faculty of Pharmacy, AIMST University, Malaysia. He obtained his Master of Clinical Pharmacy from the Science University of Malaysia and is American Association of Pharmacy Board certified Pharmacotherapy specialist as well as ambulatory care pharmacy specialist. He has been practicing cardiology pharmacy for the past 8 years before joining academy pharmacy. His research interests are in the cardiovascular system, endocrinology system and nephrology.

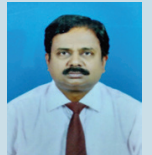

Nagula Parashuram: Obtained his MS in Otorhinolaryngology in 1994 from Kakatiya Medical College, Warangal-India. Currently working as Assistant Professor in Kakatiya Medical College, Warangal since 1999 teaching medical graduates. He has published various articles and case reports in Indian and International journals. 


\section{REFERENCES}

1. Hak AE, Pols HA, Visser TJ, Drexhage HA, Hofman A, Witteman JC. Subclinical hypothyroidism is an independant risk factor for atherosclerosis and myocardial infarction in elderly women: the Rotterdan study. Ann Intern Med. 2000;132(4):270-8

2. Golden SH, Robinson KA, Saldanha I, Anton B, Ladenson PW. Prevalence and incidence of endocrine and metabolic disorders in the United States: a comprehensive review. Journal of Clinical Endocrinology and Metabolism. 2009;94(6):1853-78.

3. Lai CC, Tang SH, Pei D, Wang CY, Chen YL. The prevalence of subclinical thyroid dysfunction and its association with metabolic syndrome in Taiwanese Elderly. Int J Gerontol. 2011;5(1):25-9.

4. Gaurav A, Sudhakar MK, Mohini S, Senthil N, Amarbalan R. The prevalence of thyroid dysfunction among South Indian women with Metabolic Syndrome. Journal of Clinical and Diagnostic Research. 2011;5(2):213-6.

5. Uzunlulu M, Yorulmaz E. Prevalence of subclinical hypothyroidism in patients with metabolic syndrome. Endocr J. 2007;54(1):71-6.

6. Leclere J, Cousty C, Schlienger JL, Wemeau JL. Subclinical hypothyroidism and quality of life of women aged 50 or more with hypercholesterolemia: results of HYOGA study. Presse Med. 2008;37(11):1538-46.
7. Sireesha P, Pandian GB, Parashuram N. Influence on Quality of Life among Patients with Hypothyroidism. PTB Reports. 2015;1(1):24-6.

8. Bauer DC, Ettinger B, Browner WS. Thyroid function and serum lipids in older women: a population-based study. Am J Med. 1998;104(6):546-51.

9. Vijayakumar A, Kalaiselvan V, Ganesh PB, Ranvir K, Suchandra S. Role of $11 \beta$-Hydroxysteroid dehydrogenase inhibitors in metabolic syndrome and its expansion in other therapeutic options. IJPRBS. 2013;2(5):78-99.

10. Michalaki MA, Vagenakis AG, Leonardou AS, Argentou MN, Habeos IG, Makri MG. Thyroid function in humans with Morbid Obesity. Thyroid. 2006;16(1):73-8.

11. Bastemir M, Akin F, Alkis E, Kaptanoglu B. Obesity is associated with increased serum TSH level, independent of thyroid function. Swiss Med Wkly. 2007;137(29/30):141-4.

12. Stichel $H$, I'Alleman $D$, Gruter A. Thyroid function and obesity in children and adolescents. Horm Res. 2000;54(1):14-9.

13. Jayakumar RV Nisha B, Unnikrishnan AG, Nair $V_{t}$ Kumar $H$. Thyroid status in metabolic syndrome-a clinical study. Thyroid Research and Practice. 2010;7(2):47-51.

14. Reaven GM. Role of insulin resistence in human disease. Diabetes. 1988;37(12):1595-607. 